\title{
Materias preciosas textiles: el caso del bordado con posible escena de Pentecostés del Museo Nacional de Artes Decorativas
}

\author{
Ana Cabrera Lafuente \\ Museo Nacional de Artes Decorativas \\ ana.cabrera@mecd.es
}

\begin{abstract}
RESUMEN
El artículo presenta la tecnología de los hilos metálicos utilizada en los textiles de la Edad Media a partir de un fragmento de bordado conservado en el Museo Nacional de Artes Decorativas. Se explica el origen y desarrollo de la tecnología de los hilos metálicos a partir de los diferentes metales y aleaciones utilizados. Asimismo se estudian la procedencia y el taller de bordado como un ejemplo de la diversidad de modelos decorativos y talleres activos durante los siglos XII y XIII en el Mediterráneo occidental.
\end{abstract}

Palabras clave: Hilos metálicos, tecnología, bordado, cartones, talleres de bordado, influencia siculobizantina, Calahorra (La Rioja).

\section{Precious Textile Materials: The Case of the Embroidery with a Possible Pentecost Scene in the Museo Nacional de Artes Decorativas}

\begin{abstract}
This paper presents the technology of the metallic threads used in the textiles of the Middle Ages through the study of an embroidery fragment preserved in the Museo Nacional de Artes Decorativas. The origin and the development of the metallic threads technology are explained, including the different metals and alloys used. The paper also studies the questions related with the provenance and workshop of the embroidery as an example of the diversity of decorative sources and workshops active in the $12^{\text {th }}-13^{\text {th }}$ centuries in the Western Mediterranean area.
\end{abstract}

Key words: Metallic threads, technology, embroidery, cartoons, embroidery workshops, siculo-byzantine influence, Calahorra (La Rioja, Spain). 
El Museo Nacional de Artes Decorativas (MNAD) custodia entre su fondo de tejidos un interesante fragmento bordado que fue adquirido en el año 2005 en pública subas$\mathrm{ta}^{1}$. Este tejido llamó desde el principio la atención del museo al corresponderse con el publicado por Floriano Cumbreño ${ }^{2}$ en 1942 como parte de los tejidos de la catedral de Calahorra y que se daba por perdido desde la Guerra Civil.

\section{Descripción del bordado}

Se trata de un fragmento (Fig. 1) de forma casi cuadrada de $33 \mathrm{~cm}$ de alto por $23 \mathrm{~cm}$ de ancho, con importantes pérdidas y un estado de conservación muy frágil, lo que ha obligado a realizar una intervención de limpieza y encapsulado con tul ${ }^{3}$.

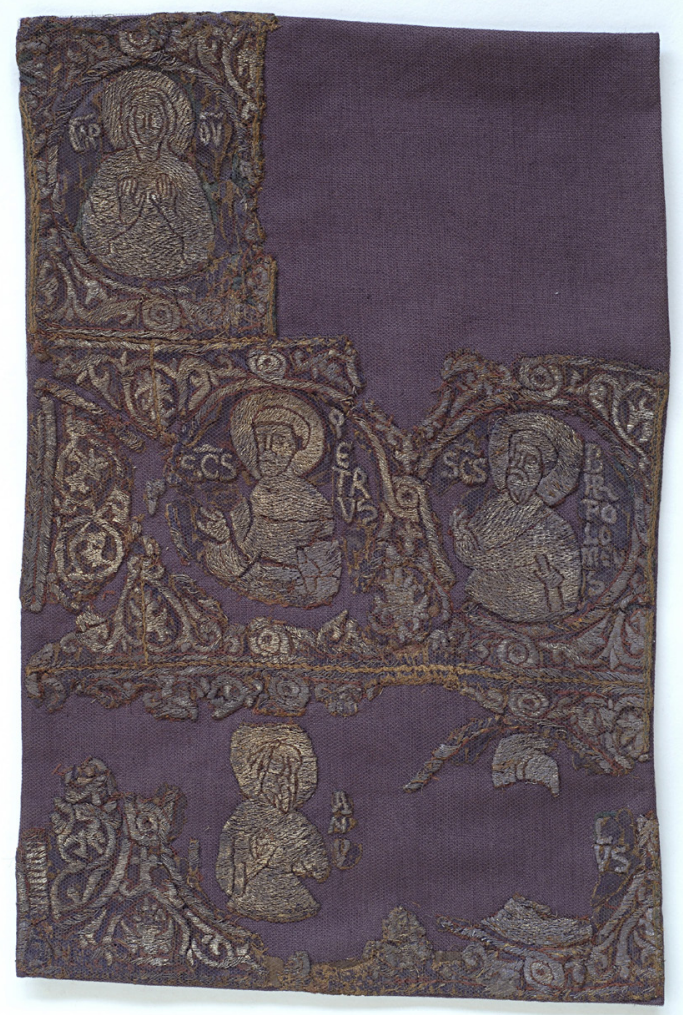

Fig. 1. Vista general del bordado MNADCE26817 (@ Museo Nacional de Artes Decorativas, Madrid. Ministerio de Educación, Cultura y Deporte).

\footnotetext{
1 Tiene como número de inventario el CE26817.

2 A. FLORIANO CUMBREÑO, El Bordado, Barcelona, 1942, pp. 53-54, fig. 11.

3 Realizada por Mónica Enamorado en el 2006. El encapsulado trata de proteger al tejido por todos sus lados, impidiendo más pérdidas e inmovilizándolo.
} 
El tejido está totalmente cubierto por la decoración bordada mediante hilos metálicos dorados y de seda. La decoración se organiza en tres franjas y se compone de cinco figuras, la quinta muy perdida, en medallones realizados mediante un tallo liso con nudos abiertos en los ejes vertical y horizontal. En la franja superior aparece una figura velada con las manos en actitud orante. En la segunda dos figuras barbadas mirando hacia el centro y con un objeto o rollo en su mano izquierda. En la última franja otra figura barbada mirando hacia la derecha y sosteniendo un rollo con la mano diestra. La última figura está muy perdida, aunque se intuye el nimbo, elemento que caracteriza a todas las figuras.

Las figuras están bordadas a hilos tendidos con hilos metálicos de plata dorada y los medallones están separados cada uno por una franja de roleos realizada por una fila triple de hilos tendidos de seda. Todos los hilos están sujetos por un hilo de seda (Fig. 2).

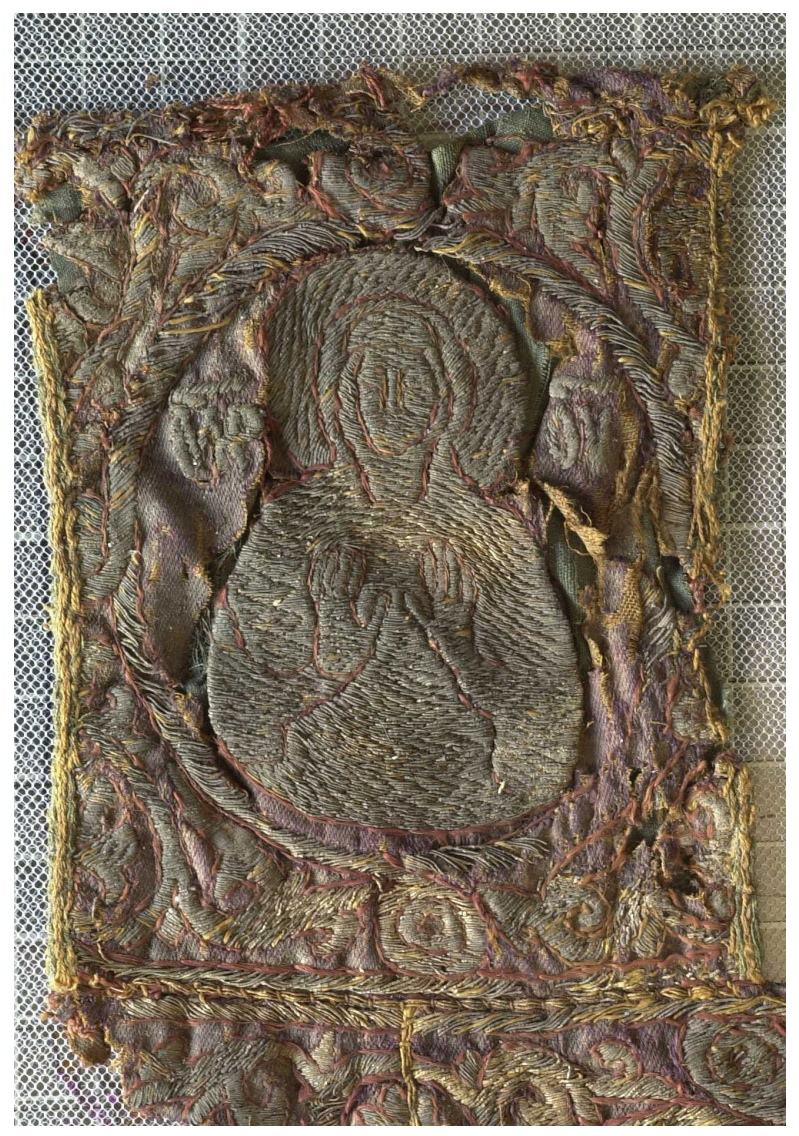

Fig. 2. Detalle de la figura de la Virgen orante. En la parte superior se pueden apreciar las dos capas de tejidos, el tafetán de seda verde y la sarga de color morado (MNADCE26817, (C) Museo Nacional de Artes Decorativas, Madrid. Ministerio de Educación, Cultura y Deporte). 
Cada figura está identificada por unos tituli, que de arriba abajo y de izquierda a derecha son:

- MP- $\Theta Y$

- SCS-P-E-TR-US

- SCS B-AR-TO-LO-MEU-S

- ... A-N-U..

- ....L-US

La lectura de las inscripciones completas sería $M[\dot{\eta} \tau \eta] \rho \Theta[\varepsilon o \eta] \chi$, S[anctu]s Petrus y $S[a n c t u] s$ Bartolomeus. La primera corresponde a la denominación de Madre de Dios en griego, mientras que las otras dos son los nombres de los apóstoles Pedro y Bartolomé, los cuales llevan el rollo aludiendo a su misión de extender la palabra evangélica. La lectura de las inscripciones incompletas podría interpretarse como los nombres de otros apóstoles, Pablo (Paulus) o Juan (Ioannus).

La interpretación de las mismas vendría dada por la explicación de este tipo de escenas, que ya Cumbreño identifica como de una representación de Pentecostés, por lo que el resto de las figuras serían los demás apóstoles. Tampoco puede descartarse que fuera una representación de la Ascensión o de la Virgen con distintos santos. Estas últimas tendrían en la parte superior escenas de la Biblia, tal y como aparece en los marfiles y esmaltes, especialmente el díptico de Constantinopla conservado en la Biblioteca Nacional de Francia, tal y como señaló $\mathrm{M}^{\mathrm{a}}$ Dolores Vila ${ }^{4}$.

Tanto si es la Ascensión como Pentecostés, su iconografía es muy antigua, remontándose las primeras representaciones al Evangeliario sirio de Rabula del año $587^{5}$ que muestran una de las variantes de esta escena con la Virgen en el centro de la misma, como podría ser nuestro caso ${ }^{6}$. Tampoco puede descartarse que se trate de un Apostolado presidido por la Virgen, como aparece en los iconostasios de las iglesias bizantinas.

\section{Técnica de fabricación}

A un nivel general el bordado está montado sobre dos capas de tela. La primera, un tafetán de seda verde que hace las veces de soporte a una sarga de color morado, la segunda capa textil, que está totalmente cubierta del bordado (Fig. 2).

La técnica de hilos tendidos consiste en desplegar los hilos siguiendo el diseño de la decoración y se sujetan con pequeñas puntadas a la base. Esta técnica es conocida desde la Antigüedad, aunque los mejores ejemplos que tenemos son de época medieval.

4 M.D. VILA, Ponferrada: templarios, peregrinos y señores: el mundo de los castillos, Valladolid, 2010, p. 178.

5 Folios 13v. (Ascensión) y 14v. (Pentecostés). H.L. KESSLER, “The Word made Flesh in Early Decorated Bibles", J. SPIER (ed.), Picturing the Bible. The Earliest Christian Art, catálogo de la exposición, New Haven, 2007, pp. 164-166 y cat. no 82, pp. 276-282.

6 L. RÉAU, Iconografia del arte cristiano. Iconografia de la Biblia. Nuevo Testamento, t. I, vol. II, Barcelona, 1996, pp. 604-618. 


\begin{tabular}{|l|r|r|r|r|}
\hline \multicolumn{1}{|c|}{ ZoNA ANALIZADA } & $\mathbf{A g}$ & $\mathbf{C l}$ & $\mathbf{C u}$ & $\mathbf{A u}$ \\
\hline Hilo 1 base exterior & 74,0 & 26,0 & & \\
\hline Hilo 1 restos dorado exterior 1 & 38,2 & 10,9 & 3,1 & 47,8 \\
\hline Hilo 1 restos dorado exterior 2 & 24,6 & 5,4 & 2,9 & 67,1 \\
\hline Hilo 1 restos dorado exterior 3 & 27,3 & 6,8 & 2,3 & 63,6 \\
\hline Hilo 1 restos dorado exterior 4 & 33,9 & 7,7 & 2,1 & 56,4 \\
\hline Hilo 2 base exterior & 75,7 & 24,3 & & \\
\hline Hilo 2 base interior 1 & 96,8 & 3,2 & & \\
\hline Hilo 2 base interior 2 & 87,8 & 12,2 & & \\
\hline Hilo 2 dorado exterior 1 & 43,4 & & 2,3 & 54,3 \\
\hline Hilo 2 dorado exterior 2 & 38,2 & 2,0 & 2,2 & 57,6 \\
\hline
\end{tabular}

Tabla 1. Composición (en \%) de los hilos metálicos analizados. Es notable la presencia de cloruros y la detección del oro $(\mathrm{Au})$ en una sola cara.

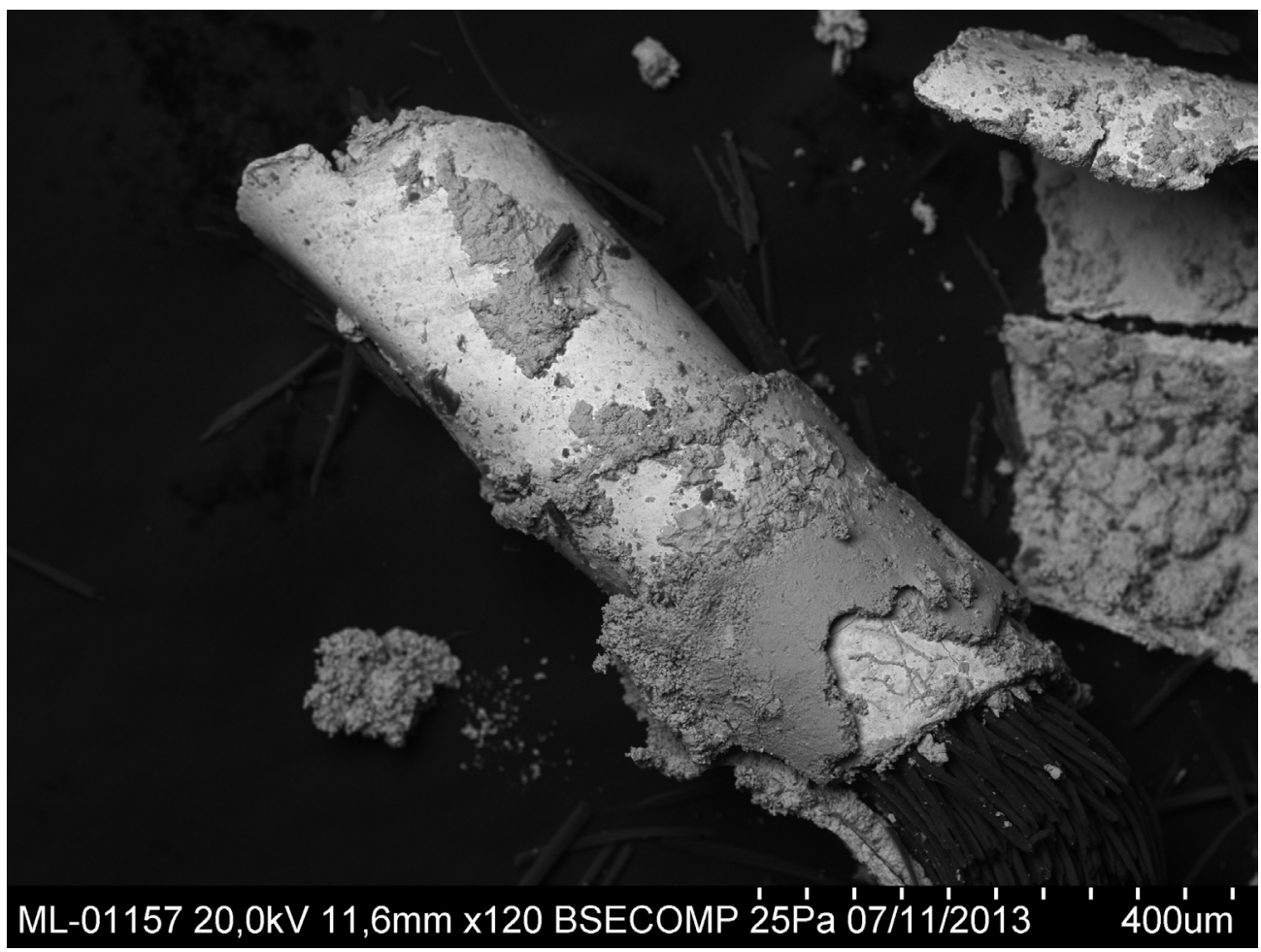

Fig. 3. Fotografía del microscopio electrónico en donde se puede ver la capa de sulfuros del hilo de muestra analizado y cómo la suciedad se acumula en las fibras (Microlab del CSIC, fotografía realizada por Oscar García-Vuelta). 
Los hilos metálicos usados son de plata dorada por una sola cara, la visible (Tabla $1)^{7}$. La presencia de cobre en la aleación de plata ha dado lugar a un serio problema de conservación al estar los hilos metálicos atacados por sulfuros, producto de la descomposición de estos metales, escamando la superficie ${ }^{8}$ (Fig. 3) y haciendo que el tejido tenga ese aspecto de suciedad generalizada. Esta corrosión ha hecho perder el aspecto original del tejido que sería de un cierto impacto, al estar las figuras en dorado y los medallones silueteados por hilos de seda, lo que haría fijar la vista en las figuras. El contraste sería muy parecido a ciertas piezas de metalistería.

Dada la importancia de los hilos metálicos en los tejidos de este periodo tanto en Occidente como Oriente, merece la pena detenernos más en este tipo de manufactura.

\section{Hilos metálicos: historia y técnicas de fabricación}

Las primeras referencias que tenemos sobre el uso de los metales preciosos aplicados a tejidos es la descripción de las vestiduras del Sumo Sacerdote judío recogida en el libro del Éxodo, en ella se describe el ephod, o túnica, tejido con hilos de oro y de color azul, púrpura y rojo, realizada en lino y bordada en hilo de oro 9 . Otro texto judío, el Talmud, recoge que estaba hecho con un ligamento de seis hilos, siendo el séptimo de una lámina de oro.

Las fuentes griegas, tanto literarias como la Odisea o autores como Herodoto, describen vestiduras adornadas con hilos de oro ${ }^{10}$. Las latinas, por su parte, con Plinio el Viejo a la cabeza, hacen también abundantes alusiones a ropas y telas con hilos metálicos de oro, cuya denominación en latín sería, según las fuentes, la de auratae vestes.

Este tipo de tejidos suelen ser descritos como telas o vestidos de seda de color púrpura o teñidos con púrpura e hilos dorados, y son por tanto los tejidos más caros y lujosos. La razón de su elevado precio se debe al hecho de estar manufacturados con tres de las materias primas más caras de la Antigüedad: el tinte púrpura, la seda y los hilos metálicos. Debían ser tan caros que Marco Aurelio financió parte de su campaña militar vendiendo los vestidos de seda y de oro de su mujer. En el Edicto de Precios Máximo de Diocleciano de 301 d.C., el precio o salario para los tejedores de los tejidos en seda e hilo de oro era de 1.000 denarios, el más alto de todos ${ }^{11}$.

\footnotetext{
7 Los análisis han sido realizados en el Microlab del CSIC por Oscar García-Vuelta e Ignacio Montero, a los que estoy muy agradecida por las explicaciones, las imágenes y la resolución de cualquiera de mis dudas.

8 La corrosión de la plata afecta a la seda, al depositarse los sulfuros sobre los hilos. En los hilos de tejidos de terciopelo estudiados por Ferrrazza y Juanes observan que los hilos metálicos con la superficie sulfatada se podría relacionar con una capa de dorado irregular, lo que confiere un aspecto oscuro en la superficie: L. FERRAZZA y D. JUANES, "Análisis e identificación de fibras, mordientes e hilos entorchados metálicos", L'Art dels Velluters. Sederias de los siglos XV-XVI, Valencia, 2011, pp. CXIX-CXXII.

9 Éxodo, 28: 6-14.

10 Un resumen de las referencias y de hallazgos arqueológicos de hilos metálicos se encuentra en M. GLEBA, "Auratae vestes: Gold textiles in the ancient Mediterranean", C. ALFARO y L. KARALI (eds.), Vestidos, textiles y tintes. Estudios sobre la producción de bienes de consumo en la Antigüedad. Purpureae Vestes, II, Valencia, 2008, pp. 61-77.

11 Ibid, p. 62.
} 
El gran número de referencias en las fuentes escritas de la Antigüedad a este tipo de tejidos no es paralelo al número de tejidos encontrados con estas características, ni a una descripción minuciosa de cómo se hacían, en qué talleres y qué artesanos eran responsables de su manufactura.

En los escritos romanos aparecen mencionados chryso-phygium, phrygiae vestes $\mathrm{y}$ opus phrygionicum además de barbaricae vestes phrygiae. Estos nombres indican no solo que se trata de tejidos con hilos de oro, también parecen indicar el origen de la tecnología y su lugar de fabricación en Oriente Próximo. Este origen podría demostrarse también en los escasos hallazgos arqueológicos, pues los orientales (Siria, Ucrania, Rusia $^{12}$ ) son más tempranos que los occidentales.

En cuanto a quienes lo fabricaban, las escasas fuentes escritas hablan de joyeros ${ }^{13}$, mientras que la epigrafía recoge nombres como aurinetrix y aurivestrix, mujeres especialistas en el hilado del hilo de oro y en la venta de las auratae vestes respectivamente. Otras hablan de barbaricarii, productores de tejidos con hilos de oro y plata. En el s. IV d.C. en el Código de Teodosio los barbaricarii de Constantinopla y Antioquía se habían organizado en fábricas que suplían a los círculos imperiales ${ }^{14}$. Entre el $\mathrm{s}$. IV y el VI d.C. la manufactura de los tejidos en seda e hilos de oro se convirtió en monopolio del Estado romano y los artesanos o fábricas de particulares no podían hacerlos; este sistema era muy similar en el imperio Bizantino con el taller palatino.

Con todo, sí parece que la fabricación de hilos metálicos estaba plenamente estructurada y que podían ser manufacturados tanto por joyeros como por otros artesanos. Estos últimos tenían los distintos estadios de su producción diferenciados en los que preparaban las láminas de oro, los que los hilaban, los que los tejían y los que los vendían. Este tipo de organización se mantiene por lo menos hasta el s. VI d.C. en Occidente, mientras que en el Imperio Bizantino duraría más tiempo, en relación con la producción del taller palatino.

Y, ¿cómo eran los hilos metálicos? Por los tejidos encontrados podemos dividirlos en cinco tipos:

- Hilo metálico de sección circular, descrito en las fuentes como alambre ${ }^{15}$.

- Lámina metálica.

- Alambre arrollado a un hilo, normalmente de seda.

- Lámina arrollada a un hilo de fibra textil.

- Membrana dorada arrollada a un hilo de fibra textil.

En la Edad Media seguimos encontrado gran número de fuentes escritas que hablan de las ricas telas en seda e hilos de oro y plata pero escasas referencias a su modo de fabricación. Pero, al contrario que en la Antigüedad, de la que tenemos pocos ejemplares, en la Edad Media se han conservado un número mayor de tejidos con hilos metálicos.

\footnotetext{
12 Tanto Ucrania como Rusia formaban parte del territorio escita y sármata, con colonias griegas y posteriormente romanas en el Mar Negro y con relaciones comerciales con China.

13 Demóstenes en uno de sus discursos habla de su himation dorado producido por el joyero Pamenides para llevarlo en la procesión dionisiaca.

14 Cod. Theo., 10.22.1

15 M. GLEBA, op. cit., 2008, p. 68, habla de "gold wire".
} 
Estos hilos se corresponden tipológicamente con los dos últimos enumerados más arriba. El que más perdurará será el hilo o lámina arrollada a un alma de fibra, que continúa durante la Edad Moderna, mientras que el hilo de membrana dorada tendrá su momento en la Edad Media.

Además, en este periodo se documentan una serie de cambios en la técnica de manufactura de los hilos metálicos ${ }^{16}$, al conseguir una mayor longitud de las láminas, la aparición de hilos con láminas de plata dorada y los hilos de membrana dorada.

La mayor longitud de las láminas que se arrollan a un alma de fibra es notable, ya que en los casos estudiados llega a alcanzar varios kilómetros, como en la capa de Santa Cunegunda conservada en la Catedral de Bamberg (Baviera, Alemania) ${ }^{17}$. La posibilidad de conseguir una mayor longitud en las láminas demuestra el mayor control y desarrollo en la fabricación de estos tipos de hilos. Esta técnica podría ser la descrita por el monje Teofilus en el s. XII ${ }^{18}$, con el estiramiento de los alambres obtenidos mediante el organarium $^{19}$. Para tener una idea del nivel técnico alcanzado basta conocer el diámetro de los alambres usados, que era de alrededor de 0’05 $\mathrm{mm}$.

La composición de los hilos metálicos va variando a lo largo del tiempo en la Edad Media. Al principio será con un alto porcentaje de oro, pero cada vez será menor su cantidad, dado su valor. El caro precio del oro sería una de las razones que posiblemente desarrollasen los hilos de plata dorada por una sola cara, que ya aparecen en tejidos del s. $\mathrm{X}^{20}$ y se extiende en Europa entre los siglos XII y XIII. Es interesante observar que estos hilos se usan mayoritariamente para bordar ${ }^{21}$.

De nuevo es el monje Teofilus quien describe la técnica empleada para estos hilos, que consistía en la forja de una pieza rectangular de plata que se cubría de oro soldándose ambos metales con cobre y otros materiales de soldadura. En nuestro caso la lámina de plata tiene un grosor medio de 17 micras (véase la finura de la capa dorada en la Fig. 4). Teofilus comenta que la proporción entre ambos metales sería 1 parte de oro por 12 partes de plata, con el consiguiente abaratamiento de costes. Este tipo de hilos se seguirían usando en el s. XVI.

La tercera innovación que se produce en este periodo es la aparición de un nuevo tipo de hilo hecho mediante la aplicación de una hoja o pan de oro a una membrana o piel de origen animal ${ }^{22}$. Estos hilos eran los más baratos de todos al usar, si cabe, me-

16 M. JARÓ, E. GONDAR y A. TOTH, "Technical revolutions in producing gold threads used for European textile decoration", Outils et ateliers d'orfèvres des temps anciens, Saint Germain-en-Laye, 1993, pp. 119-124.

17 M. JARÓ, "Gold embroidery and Fabrics in Europe: XI-XIV centuries", Gold Bulletin, 23, 2 (1990), p. 46.

18 Este monje benedictino, posiblemente trabajador de los metales, es identificado por Whitfield con Roger de Halmarshausen y es conocido por su obra Schedula diversarum Artium.

19 M. JARÓ, E. GONDAR y A. TOTH, op. cit., 1993, fig. 8, p. 121. Para saber más sobre estas herramientas vid. N. WHITFIELD, "The manufacture of the beaded wire in the post-Roman Period", A. PEREA, I. MONTERO y O. GARCÍA-VUELTA (eds.), Tecnología del oro antiguo: Europa y América. Anejos de Archivo Español de Arqueología, XXXII (2004), pp. 127-139.

20 M. JARÓ, E. GONDAR y A. TOTH, op. cit, 1993, p. 121.

21 Quizás estén relacionados con el apogeo del Opus Anglicanum.

22 Al principio serían todos de pan de oro, pero según pase el tiempo serían de plata dorada. 
nor cantidad de metal precioso y también los más ligeros ${ }^{23}$. La procedencia oriental de estos hilos lo recoge su propia denominación histórica, ya que las fuentes hablan de "hilos de Chipre" 24.

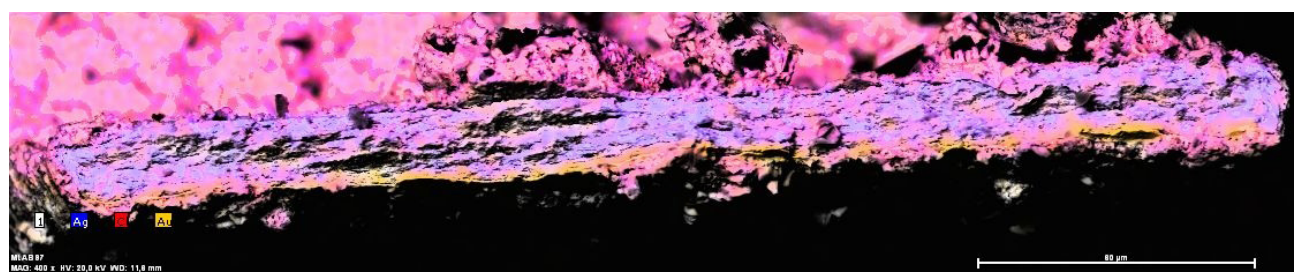

Fig. 4. Fotografía de un corte del hilo metálico con la composición. La capa inferior, que sería la externa del hilo, presenta el dorado de oro, el centro es de plata y el resto es de cobre (Microlab del CSIC, fotografía y esquema realizados por Oscar García-Vuelta).

En la Península Ibérica estos hilos son los que aparecen en las tapicerías andalusíes como la Franja del Pirineo o el Almaizar de Hisam II. Las fuentes medievales islámicas describen también quiénes y dónde se hacían este tipo de hilos. En algunos casos en los talleres platinos o tiraz, en otros eran los dhahabi, joyero y fabricante de hilos de oro, tal y como lo recoge Shatzmiller en su libro sobre el trabajo en el mundo islámico ${ }^{25}$. Otro término relacionado es el de zarkash, bordado en oro y plata. Todo ello muestra cómo la tecnología de fabricación era conocida, además de en Oriente, en todo el ámbito mediterráneo y europeo.

Los estudios de los años 90, especialmente por parte de Martha Jaró, documentan que estos hilos en un principio usaban cuero de una gran finura, y que a partir del s. XIII la parte orgánica cambia y, en vez de cuero, se empieza a utilizar una fina membrana orgánica (quizás tripa) y, además, el metal usado en el pan no será oro, sino plata dorada ${ }^{26}$.

Otro procedimiento, que podría relacionarse con el alto coste de estos tejidos preciosos, es que en algunos tejidos ya más tardíos o de época moderna el hilo metálico no cubre totalmente el alma o hilo de fibra textil, dejándolo al descubierto ${ }^{27}$. En este caso la fibra es de seda y suele estar teñida de amarillo, como ocurre en algunos de los tejidos nazaríes.

23 El ejemplo descrito por M. Jaró es claro, se trata del manto de coronación húngaro, bordado en hilos de oro de gran pureza que supone unos 4'4 kg de este metal. Con esta técnica el total del oro usado pesaría 0’3 $\mathrm{kg}$ (M. JARÓ, E. GONDAR y A. TOTH, op. cit, 1993, p. 123)

24 A este tipo de hilo se le llamaba también oropel en los documentos medievales europeos: C. PARTEARROYO LACABA, "Tejidos andalusíes", Artigrama, 22 (2007), pp. 371-419, y "Estudio históricoartístico de los tejidos en al-Andalus y afines", Bienes Culturales, 5 (2005), p. 42.

25 M. SHATZMILLER, Labour in medieval Islamic world, Nueva York, 1994, p. 123.

26 M. JARÓ, E. GONDAR y A. TOHT, "Reconstruction of gilding techniques used in medieval membrane threads in museum textiles”, E. PERNICKA y G.A. WARNER (eds.) Archaeometry’90, Basilea, 1990, pp. 320-322.

27 Rinuy denomina a los hilos metálicos que cubren todo el alma como cerrados, mientras los que dejan ver el alma los llama abiertos: A. RINUY, “Analyse des Goldfäden”, A. SCHMIDT-COLINET, A. STAUFFER y K. AL-AS'AD (eds.), DieTextilien aus Palmyra, Neue und alte Funde, Mainz-am-Rhein, 2000, p. 17. 
Con todo, parece claro que la Edad Media fue un momento de innovación y desarrollo en los tejidos en general, con la evolución de los ligamentos desde tejidos simples (sarga, tafetán, tapicería) a otros más complejos (taqueté, samito, lampas... acabando con el terciopelo) y, por supuesto, los hilos metálicos. El conocimiento de las distintas tipologías ayuda a la hora de clasificar y datar los tejidos, como es el caso que nos ocupa.

El bordado del MNAD está realizado con hilos de plata dorada a una cara que nos remiten a producciones de los siglos XII y XIII. Más difícil es saber su lugar de producción. Cumbreño lo enmarca dentro de las producciones españolas "de supervivencia bizantina dentro del románico" y del s. XIII" ${ }^{28}$.

En nuestra opinión tampoco podría descartarse, por la técnica empleada y los paralelos localizados, que se trate de un bordado del Sur de Italia y de influencia bizantina, siendo el paralelo más cercano la mitra del Tesoro de la Catedral de Capua ${ }^{29}$. Ambas piezas son similares tanto en la técnica del bordado, bastante común, por lo que no es muy indicativa del lugar de producción, como en el tipo de tejidos usados para la base (tafetán y sargas de seda), además de que las figuras son muy similares en su dibujo y frontalidad, especialmente la Virgen.

En la pieza de Capua sorprende que en los títulos de los dos apóstoles representados (Pablo y Pedro) la $S$ de "Sanctus" esté en latín y el nombre en griego. Como recoge Guastella, este tipo de trabajo estaría relacionado con un centro local del Sur de Italia, pero de una cultura no exclusivamente griega ${ }^{30}$. Todo ello la lleva a considerar la mitra capuana como una pieza de alta calidad dentro del círculo constantinopolitano pero realizada en un centro occidental, en donde la cultura bizantina había alcanzado el nivel del refinamiento de la corte, posiblemente en relación con la corte tardo normanda-siciliana por la mezcla de elementos, especialmente en los tituli. La autora italiana comenta que este tipo de piezas ${ }^{31}$ se realizarían en un taller de bordado de alto nivel formal y técnico en el que las decoraciones serían comunes, pero donde la línea y expresividad de las figuras dependería de los distintos maestros o dibujantes de los cartones, de la calidad del bordador y de sus distintas procedencias. Tanto el bordado como el diseño de los cartones estaban en manos de los monjes o de artesanos especializados.

Este tipo de producción pone de relieve la importancia de los cartones usados en el diseño del bordado. En general, los tejidos necesitan de un diseño previo o cartón para su realización. En este caso la mezcla de lenguas en los tituli podría deberse al uso de dos tipos de cartones (uno bizantino y otro latino), o a que el diseñador del cartón (normalmente un monje) tenía acceso a obras bizantinas y a piezas con este

28 A. FLORIANO CUMBREÑO, op. cit, 1942, p. 53 y cuadro II: "El bordado español. Sinopsis cronológica".

29 Esta mitra se atribuye a San Paulino, está realizada en seda, con un bordado de hilos de oro, plata y sedas polícromas: C. GUASTELLA: "La mitra detta di San Paolino", M. ANDALORO (ed.), Federico e la Sicilia. Dalla terra alla corona, catálogo de la exposición, Palermo, 2000, fig. 15.1, pp. 101-103.

30 Ibid., p. 101.

31 Incluye, además de la mitra de Capua, la mitra de Anagni, donada por el Papa Bonifacio VIII: ibid., p. 102. 
tipo de representaciones ${ }^{32}$, o que se trate de monjes con distinta cultura o procedencia, como ocurre con el alba del Tesoro Imperial de Viena, que conserva en el reverso de los bordados de la parte superior de los brazos y de uno de los puños las firmas de los bordadores y ha demostrado la colaboración entre maestros de distinto origen ${ }^{33}$.

Otro punto interesante es su procedencia, ya que el tejido no es el único que tiene vinculación con Bizancio entre los objetos de arte procedentes de Calahorra. La National Gallery de Washington conserva dos pinturas sobre tabla, la Madonna Mellon y la Madonna Khan que están catalogadas como procedentes de un monasterio de Calahorra. Ambas obras son bizantinas de la misma cronología que nuestra obra ${ }^{34}$. El hecho de que en Calahorra hubiera otras obras bizantinas del mismo periodo podría apoyar la hipótesis de que este bordado procediera de un taller sur-itálico. Esta obras no son las únicas con esta procedencia, las importantes relaciones del reino de Aragón con Bizancio y el Sur de Italia son buena prueba de ello ${ }^{35}$.

En conclusión, este pequeño fragmento es una buena muestra de las denominadas materias textiles preciosas, como lo atestigua la cantidad de seda e hilos metálicos de plata dorada con los que este bordado está manufacturado. Creemos que puede servir como ejemplo de la información que de los tejidos se puede obtener, documentando las materias primas y técnicas empleadas, así como su decoración, que podrían acercarnos a la compleja realidad de las relaciones entre la Península Ibérica y el Mediterráneo en los siglos XII y XII.

32 Una de las más similares a la representación de la Virgen es el pequeño icono bizantino con la Virgen Orante de la Walters Art Gallery (inv. 57.1818), en plata dorada y datada en el s. XII (http://art.thewalters. org/detail/3379/icon-of-the-virgin/, última consulta 15/4/2014). Agradezco a la Dra. C. Cosmen y a Noemí Salazar, de la Universidad de León que me comentaran esta pieza.

33 Kunsthistorisches Museum Viena. The secular and Ecclesiastical Treasuries, Viena, 1991, cat. $\mathrm{n}^{\circ} 144$, pp. 139-142). También se puede consultar en http://www.google.com/culturalinstitute/asset-viewer/the-alba/ iwHQr37djBjMtA?projectId=art-project (última consulta 15/4/2014).

34 Agradezco al Dr. Manuel Castiñeiras su amable comentario a este respecto. Ambas pinturas presentan dudas sobre su procedencia, siendo más discutida la Madonna Mellon, ya publicada por Berenson como procedente de Calahorra: B. BERENSON, "Due dipinti del decimo secondo secolo venuti da Costantinopoli", Dedalo, 2 (1921), pp. 284-304. La Madonna Khan aparece ya documentada en 1912 en la colección de Herbert P. Weissberger, donde se dice que procede de una iglesia o convento de Calahorra. Weissberger era un conocido coleccionista y marchante de arte que colaboró en varias de las exposiciones organizadas por la Sociedad de Amigos del Arte.

35 Sirva como muestra Bizancio en España. De la Antigüedad tardía a El Greco, catálogo de la exposición, Madrid, 2003. 